\title{
1.
The Implications of Significance Testing Based on Hypothesiswise and Studywise Error
}

\author{
Mark Rubin \\ The University of Newcastle, Australia
}

Citation: Rubin, M. (2017). The implications of significance testing based on hypothesiswise and studywise error. PsycArXiv. doi: 10.17605/OSF.IO/7YFRV Retrieved from https://psyarxiv.com/7yfrv/

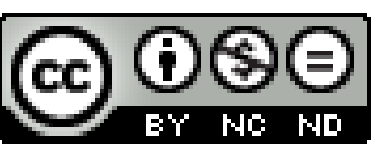

Copyright $(9)$ The Author. OPEN ACCESS: This material is published under the terms of the Creative Commons Attribution-NonCommercial-NoDerivatives 4.0 International licence (CC BY-NC-ND 4.0; https://creativecommons.org/licenses/bync-nd/4.0/). This licence permits you to copy and redistribute this material in any medium or format for noncommerical purposes without remixing, transforming, or building on the material provided that proper attribution to the authors is given.

The material in this article has not undergone pre-publication peer review. Post-publication peer review is welcome via outlets such as ResearchGate, $\underline{\text { Publons, }}$, and PubPeer.

Correspondence concerning this article should be addressed to Mark Rubin at the School of Psychology, Behavioural Sciences Building, The University of Newcastle, Callaghan, NSW 2308, Australia. Tel: +61 (0)2 4921 6706. Fax: +61 (0)2 4921 6980. E-mail: Mark.Rubin@newcastle.edu.au Twitter: @Rubinpsyc Web: http://bit.ly/QgpV4O

I am grateful to Serje Robidoux, Max Colheart, Teresa Schubert, Vince Olito, and other members of the Everything is Fucked (EisF) group for taking the time to discuss my Rubin (2017) paper. I am also grateful to Robidoux (2017) for his recent blog post summarising the EisF group's comments and concerns. To recap, my Rubin (2017) paper points out that familywise error can be operationalized in two ways. The first way is to consider the probability of incorrectly rejecting a joint nil null hypothesis that the overall effect predicted by all of the hypotheses tested in the study is zero. I call this the studywise error probability. The greater the number of hypotheses in a study, the higher the studywise error probability, because there are a greater number of opportunities to incorrectly reject the studywise joint null hypothesis. Following Matsunaga (2007), the second way to operationalize familywise error is to consider the probability of incorrectly rejecting each single individual null hypothesis separately. Here, I call this the hypothesiswise error probability. The greater the number of tests that are used to test a single hypothesis, the higher the hypothesiswise error probability, because there are a greater number of opportunities to incorrectly reject the associated null hypothesis. In my paper, I argue that studywise error becomes unknowable in exploratory analyses because it is unclear how many hypotheses have or could be tested. However, hypothesiswise error remains knowable because it is easier to keep track of the different tests of a single hypothesis, and it is relatively obvious when logical commonsense tests have been excluded from a group of tests of a single hypothesis, especially when researchers are 
transparent about their measures (which is assisted by making materials and data available online). In discussing these ideas, Robidoux (2017) considered two examples. I discuss each in turn.

\section{Selecting a Hypothesis for Significance Testing}

In Robidoux's (2017) first example, a word recognition researcher plots out various effects in her data without conducting any significance tests. She finds that one of the plots suggests a significant interaction between major of study (Arts/STEM) and word frequency (high/low) in predicting word recognition response times. From the plot, it looks like there might be an interaction effect in which STEM students are more sensitive than Arts students to word frequency. On this basis, the researcher proceeds to conduct a significance test to see if this interaction effect is significant. In this situation, Robidoux argues that "if you've already looked at the data somehow, then Rubin's argument no longer applies. $\alpha$-level adjustments are not objectively verifiable, and so there is limited value in doing them."

I disagree with the conclusion for this particular example. I think that whether or not you need to adjust your alpha level depends on whether you define the familywise error probability in terms of studywise error or hypothesiswise error. If you define the familywise error probability in terms of studywise error, then you must be doing so because you are interested in testing the studywise joint null hypothesis that the overall effect of all of your study's hypotheses is zero. In this case, you do need to adjust your alpha level because your test of the interaction effect represents only one of multiple potential tests of the joint null hypothesis, and you need to compensate your alpha level for all of the opportunities that you have to reject the joint null hypothesis in a long run of exact replication of that study (Gelman \& Loken, 2013). However, it is not possible to adjust your alpha level because, in exploratory research, there is no way of computing how many hypothesis tests are possible (e.g., de Groot, 1956/2014).

However, studywise hypotheses rarely have any theoretical meaning, and in those cases in which they do have some theoretical meaning (e.g., every hypothesis in a study is derived from the same theory and predicts an effect with the same sign), it is usually inappropriate to test studywise hypotheses vis-à-vis tests of specific individual hypotheses. To explain, the concept of studywise error assume that the conventional alpha level (i.e., $\alpha=.05$ ) for tests of individual null hypotheses need to be lowered (e.g., to $\alpha=.005$ ) in order to maintain the alpha level for the studywise null hypothesis at the conventional level $(\alpha=.05)$ because multiple tests of the studywise null hypotheses provide multiple opportunities to reject it, thereby inflating the studywise error probability. Following this logic, once the alpha level for tests of individual null hypotheses has been adjusted, it would be appropriate to accept a significant result (e.g., $p=.001$ ) in relation to any one of the individual null hypotheses as sufficient grounds for rejecting the studywise null hypothesis at its prespecified alpha level ( $\alpha=.05$; Weber, 2007). But I do not believe that researchers want to test hypotheses in this one-hit-sufficient manner, especially when they consider studywise hypotheses or theories that are composed of different hypotheses, each with its own theoretical rationale. To illustrate, imagine that Theory X contains Hypotheses A, B, and $\mathrm{C}$, and that a researcher conducts a single test of each of these hypotheses in a single study. In this case, the studywise joint nil null hypothesis is that Theory X's hypothesised effects are equal to zero, and tests of each constituent null hypothesis (i.e., A, B, \& C) represent multiple tests of this studywise hypothesis. In this case, the alpha level for each constituent hypothesis could be lowered to .015 in order to maintain the alpha level for the studywise hypothesis at .05. However, if a researcher obtained a significant effect in relation to Hypothesis A at the adjusted alpha level (e.g., $p=.001$ ), then it is unlikely that they would then go on to reject the nil null studywise 
hypothesis that Theory $\mathrm{X}$ is false. It is more likely that the researcher would want to test Hypotheses A, B, and C separately, at their own unadjusted alpha levels (i.e., $\alpha=.05$ ), in order to draw separate conclusions about the support for each. This approach would still allow the researcher to draw conclusions about Theory X. However, these conclusions would not be based on a statistical test of Theory $\mathrm{X}$, and so they would not require an alpha adjustment. Instead, they would only be based an amalgamation of conclusions based on statistical tests of Hypotheses A, $\mathrm{B}, \& \mathrm{C}$.

To be clear, following Matsunaga (2007) and Weber (2007), I do believe that familywise error needs to be taken into account when testing hypotheses. But I think that it only needs to be computed for multiple tests of a null hypothesis when a significant result on any of those multiple tests is regarded as being sufficient to reject that hypothesis. This is not the approach that researchers take when considering studywise hypotheses or theories. Consequently, I think that researchers are more interested in hypothesiswise error than studywise error.

In Robidoux's (2017) example, the researcher is interested in testing the degree major by word frequency interaction effect. This represents a separate hypothesis from other hypotheses because it is based on a separate theoretical rationale that explains why degree major interacts with word frequency to predict response times. Consequently, this hypothesis can be tested at its own individual alpha level (e.g., $\alpha=.05$ ), regardless of whether or not it is tested alongside many other different hypotheses.

Robidoux (2017) argued that significance testing is not valid for the interaction effect hypothesis because this hypothesis was selected by the researcher from among many other potential hypotheses on the grounds that it looked "promising." Again, if the researcher defined the familywise error probability in terms of studywise error, then this this conclusion is correct because, in this case, the selection bias skews the test of the joint null hypothesis towards a significant result, and this invalidates the test. However, if the researcher is only concerned about testing hypotheses on a case-by-case basis and controlling for each test's hypothesiswise error, then selecting the interaction hypothesis because it looks promising is not problematic because it does not inflate the prespecified probability of incorrectly rejecting the associated null hypothesis in a long run of exact replications.

To be clear, eyeballing the data and cherrypicking hypotheses that look promising (e.g., have a large effect size), does increase the probability of obtaining a low $p$ value, but this probability is separate from the alpha level. The alpha level is a threshold value that is prespecified by the researcher to indicate the highest error probability that they are willing to accept in order to behave as if the null hypothesis is false when, in fact, it is true. In theory, the alpha level is based on the researcher's judgements about the practical implications and costs of making this type of error. In practice, the alpha level is based on the conventions of research field. Either way, selecting a hypothesis because it looks promising/true/has a large effect does not inflate the alpha level because the selection process does not bias the test. Certainly, the test is more likely to yield a significant result for truer effects. But that does not mean that the test is a biased test. It simply means that the test is accurately reflecting the reality of the situation by yielding significant results for true effects.

Is it "cheating" to select hypotheses for significance testing because they have a higher probability of being true or a larger effect size? Again, if researchers are concerned about the studywise error probability, then the answer is definitely "yes" because they are selecting tests of the joint null hypothesis that are more likely to reject that hypothesis and ignoring other tests that are less likely to reject that hypothesis. Hence, they are performing a biased test. However, if 
researchers are interested in testing individual hypotheses, rather than their entire study, then the answer can be "no." As an analogy, we would not mind if a word frequency researcher consulted a meta-analysis to find out which variable was most strongly related to word frequency effects and then proceeded to focus their subsequent research on that variable. In this case, the researcher is being selective and biased in their decisions about which hypotheses to test. But we would not argue that this selectivity and bias affects the validity of their statistical tests in this area.

Note that it is possible to conceive of some less exploratory situations in which selective hypothesis testing will be problematic. For example, imagine that a researcher is investigating a theory that generates two separate but theoretically-related hypotheses: that degree major interacts with word frequency to predict (a) response times and (b) memory recall. In this case, a selection bias that results in testing the first hypothesis but not the second hypothesis would invalidate any research conclusions regarding the overall success of the theory (e.g., "The present research found convincing support for Theory A"). Note that this issue is not related to alpha levels because (a) the two hypotheses are separate in the sense that each has its own unique theoretical rationale and alpha levels, and (b) alpha levels refer to errors in statistical inference, not theoretical inference.

\section{Selecting Data to Test a Specific Hypothesis}

Robidoux (2017) also provided some simulation data in which the means and $p$ values were computed for 10,000 samples of 100 participants that were drawn from a population in which the true effect was 0 (i.e., a genuine nil null effect) and the standard deviation was 1 . This simulation shows that the $p$ values form a random uniform distribution ranging from 0 to 1 . A contingency was then added to the simulation in order to remove mean differences that were lower than a specific value $(0.1$ or 0.2$)$. This approach effectively removed the higher $p$ values from the distribution and left only the smaller $p$ values. Because the true effect is a null effect, it is possible to compute the effective alpha level (i.e., the actual probability of incorrectly rejecting the null hypothesis). This effective alpha level was higher than the nominal, prespecified alpha level of .05 .

It is important to note that the simulation example is quite different from the previous interaction hypothesis example. In the simulation example, the same hypothesis is being tested again and again in different samples (i.e., a long run of exact replications). In contrast, in the interaction hypothesis example, one hypothesis is chosen from many different hypotheses and tested. The selection bias is also different in these two situations. In the simulation, the selection bias involves restricting testing to samples in which the mean differences are larger than some criterion. This approach biases the testing of the same null hypothesis. This researcher-based selection bias is the issue that I referred to in my email to Max Colheart (personal communication, 25/09/17): As I explained, "choosing the test that looks like it will be most supportive of your hypothesis and ignoring the others represents a sort of researcher-based selection bias might be described as " $p$-hacking"" (Simmons, Nelson, \& Simonsohn, 2011). Fisher (1937) also pointed out that this sort of approach is invalid because it does not include all of the relevant effects. As Fisher explained, "in our final calculation we take account of the aggregate of the entire series of results, whether successful or unsuccessful. It would clearly be illegitimate, and would rob our calculation of its basis, if the unsuccessful results were not all brought into the account" (Fisher, 1937, p. 26). The situation is similar to that of flipping a coin 10,000 times in order to test the hypothesis that it is biased but then only considering those instances in which the coin lands heads as the basis for making a decision about the hypothesis. Note that the simulation problem goes further than that of Type I errors. If a researcher excludes data that has the potential to falsify their hypothesis (i.e., 
the small-to-zero effect sizes), then they not only increase their chances of incorrectly rejecting the null hypothesis (i.e., Type I error), but also reduce their chances of correctly accepting the null hypothesis. In other words, they lose the potential for genuine falsification.

Based on Mayo's work (e.g., Mayo \& Cox, 2006), Robidoux's (2017) solution to the simulation problem is to adjust the alpha level. But I do not think it makes sense to try to compute the error that is associated with a test that is fatally flawed (invalid). Alpha adjustment does not address the problem that there is a bias against falsification. I think that a much better solution is simply to conduct unbiased testing. In other words, include all of the effect sizes (big, small, and medium) in the test.

Again, note that the simulation example is quite different from the interaction hypothesis example. In the interaction example, the selection bias biases the choice of the hypothesis, but it does not ivaludate the test of the hypothesis. In contrast, in the simulation example the selection bias invalidates the test of the hypothesis.

\section{References}

De Groot, A. D. (1956/2014). The meaning of "significance" for different types of research. Translated and annotated by Wagenmakers, E. J., Borsboom, D., Verhagen, J., Kievit, R., Bakker, M., Cramer, A.,...van der Maas, H. L. J. Acta Psychologica, 148, 188-194. doi: 10.1016/j.actpsy.2014.02.001

Fisher, R. A. (1937). The design of experiments. Edinburgh, UK: Oliver and Boyd.

Gelman, A., \& Loken, E. (2013). The garden of forking paths: Why multiple comparisons can be a problem, even when there is no "fishing expedition" or "p-hacking" and the research hypothesis was posited ahead of time. Department of Statistics, Columbia University. Retrieved from http://www.stat.columbia.edu/ gelman/research/unpublished/p_hacking.pdf

Matsunaga, M. (2007). Familywise error in multiple comparisons: Disentangling a knot through a critique of O'Keefe's arguments against alpha adjustment. Communication Methods and Measures, 1, 243-265. doi: 10.1080/19312450701641409

Mayo, D. G., \& Cox, D. R. (2006). Frequentist statistics as a theory of inductive inference. Lecture Notes-Monograph Series, 77-97. doi: 10.1214/074921706000000400

Robidoux, S. (2017). Do p values lose their meaning in exploratory analyses? It depends how you define exploratory. Retrieved from http://serjerobidoux.blogspot.com.au/2017/10/do-pvalues-lose-their-meaning-in.html

Rubin, M. (2017). Do $p$ values lose their meaning in exploratory analyses? It depends how you define the familywise error rate. Review of General Psychology, 21, 269-275. doi: $10.1037 / \mathrm{gpr} 0000123$

Simmons, J. P., Nelson, L. D., \& Simonsohn, U. (2011). False-positive psychology undisclosed flexibility in data collection and analysis allows presenting anything as significant. Psychological Science, 22, 1359-1366. doi: 10.1177/0956797611417632

Weber, R. (2007). Responses to Matsunaga: To adjust or not to adjust alpha in multiple testing: That is the question. Guidelines for alpha adjustment as response to O'Keefe's and Matsunaga's critiques. Communication Methods and Measures, 1, 281-289. doi: $\underline{10.1080 / 19312450701641391}$

Funding

\section{Conflict of Interest}


The author declares no funding sources.

The author declares no conflict of interest. 